\title{
Structure of Symmetry Groups via Cartan's Method: Survey of Four Approaches
}

\author{
Oleg I. MOROZOV
}

Moscow State Technical University of Civil Aviation, 125993 Moscow, Russia

E-mail: oim@foxcub.org

URL: http://www.foxcub.org/ oim/

Received August 08, 2005, in final form September 29, 2005; Published online October 13, 2005

Original article is available at http://www.emis.de/journals/SIGMA/2005/Paper006/

\begin{abstract}
In this review article we discuss four recent methods for computing MaurerCartan structure equations of symmetry groups of differential equations. Examples include solution of the contact equivalence problem for linear hyperbolic equations and finding a contact transformation between the generalized Hunter-Saxton equation and the Euler-Poisson equation.
\end{abstract}

Key words: Lie pseudo-groups; Maurer-Cartan forms; structure equations; symmetries of differential equations

2000 Mathematics Subject Classification: 58H05; 58J70; 35A30

\section{Introduction}

The theory of Lie groups has greatly influenced diverse branches of mathematics and physics. The main tool of the theory, Sophus Lie's infinitesimal method [30], establishes connection between continuous transformation groups and algebras of their infinitesimal generators. The method leads to many techniques of great significance in studying the group-invariant solutions and conservation laws of differential equations [42, 21, 36, 4, 28]. Application of Lie's infinitesimal method to concrete systems of differential equations requires analysis and integration of over-determined defining systems for symmetry algebras. Additional integrations need to be done when differential invariants and operators of invariant differentiations are computed via the infinitesimal method. Also, complexity of defining systems in symmetry analysis of classes of differential equations sometimes is high enough to make the full study of all branches of classification trees very hard.

An alternative approach for studying Lie (pseudo-)groups was developed by Élie Cartan [6, $7,8]$. His theory is based on characterizing transformations from a pseudo-group by means of a set of invariant differential 1-forms called Maurer-Cartan forms. Expressions of exterior differentials of Maurer-Cartan forms in terms of the forms themselves yield the Cartan structure equations for the pseudo-group. These equations contain all information about the pseudo-group, in particular, they give all differential invariants and operators of invariant differentiations. The knowledge of Maurer-Cartan forms and differential invariants enables one to solve equivalence problems for classes of differential equations and to find mappings between equivalent equations. An important feature of the method is that it does not require integration, and allows one to find Maurer-Cartan forms by means of only differentiation and linear algebra operations.

Nowadays there are different methods for computing Maurer-Cartan forms and structure equations of symmetry pseudo-groups of differential equations. In this review paper we discuss four of these methods. We restrict our attention to symmetries of partial differential equations 
only. For applications of Cartan's method to symmetries of ordinary differential equations we refer, e.g., to $[24,25,27,17,19,3,37,11,12]$.

\section{Apriori known geometric properties of a given differential equation}

The first approach is presented in $[45,26,27,1,2,3,10,13]$. It is based on apriori known information about geometric properties of a given differential equation. We illustrate the method on the example of Liouville's equation

$$
u_{x y}=e^{u} .
$$

It is a hyperbolic equation of second order in two independent variables.

From the geometric theory of such equations (see, e.g., [3]) it follows that there exists a coframe (a collection of independent 1-forms) $\vartheta, \omega^{i}, i \in\{1, \ldots, 4\}$, on $\mathbb{R}^{5}$ with coordinates $(x, y, u, p, q)$, such that a local section $\sigma:(x, y) \mapsto(x, y, u(x, y), p(x, y), q(x, y))$ of the trivial bundle $\mathbb{R}^{5} \rightarrow \mathbb{R}^{2},(x, y, u, p, q) \mapsto(x, y)$, provides a local solution $u(x, y)$ of equation (1) whenever $\sigma^{*} \vartheta=0, \sigma^{*}\left(\omega^{1} \wedge \omega^{2}\right)=0$, and $\sigma^{*}\left(\omega^{3} \wedge \omega^{4}\right)=0$. Indeed, we take

$$
\vartheta=d u-p d x-q d y, \quad \omega^{1}=d p-e^{u} d y, \quad \omega^{2}=d x, \quad \omega^{3}=d q-e^{u} d x, \quad \omega^{4}=d y .
$$

Then a local diffeomorphism $\Phi: \mathbb{R}^{5} \rightarrow \mathbb{R}^{5}, \Phi:(x, y, u, p, q) \mapsto(\bar{x}, \bar{y}, \bar{u}, \bar{p}, \bar{q})$, is a contact symmetry of equation (1) whenever it preserves the ideal generated by the contact form $\vartheta$ and the ideals generated by the pairs $\omega^{1}, \omega^{2}$ and $\omega^{3}, \omega^{4}$ modulo the contact ideal. That is, $\Phi$ must satisfy the following condition:

$$
\Phi^{*}\left(\begin{array}{l}
\bar{\vartheta} \\
\bar{\omega}^{1} \\
\bar{\omega}^{2} \\
\bar{\omega}^{3} \\
\bar{\omega}^{4}
\end{array}\right)=\left(\begin{array}{lllll}
a & 0 & 0 & 0 & 0 \\
c_{1} & b_{1}^{1} & b_{2}^{1} & 0 & 0 \\
c_{2} & b_{1}^{2} & b_{2}^{2} & 0 & 0 \\
c_{3} & 0 & 0 & b_{3}^{3} & b_{4}^{3} \\
c_{4} & 0 & 0 & b_{3}^{4} & b_{4}^{4}
\end{array}\right)\left(\begin{array}{c}
\vartheta \\
\omega^{1} \\
\omega^{2} \\
\omega^{3} \\
\omega^{4}
\end{array}\right)
$$

with $a\left(b_{1}^{1} b_{2}^{2}-b_{2}^{1} b_{1}^{2}\right)\left(b_{3}^{3} b_{4}^{4}-b_{4}^{3} b_{3}^{4}\right) \neq 0$.

This is a standard set-up for Cartan's equivalence method [6, 7, 8, 15, 27, 37]. Procedures of this method give the Maurer-Cartan forms

$$
\begin{aligned}
& \theta^{1}=d u-p d x-q d y \\
& \theta^{2}=s_{1}\left(d p-e^{u} d y\right)+s_{2} d x \\
& \theta^{3}=s_{1}^{-1} d x \\
& \theta^{4}=s_{1}^{-1}\left(e^{-u}\left(d q-e^{u} d x\right)+s_{3} d y\right) \\
& \theta^{5}=s_{1} e^{u} d y \\
& \eta_{1}=s_{1}^{-1} d s_{1}+p d y \\
& \eta_{2}=s_{1} d s_{2}-s_{2} d s_{1}+s_{1}^{2} p d p-s_{1}^{-1}\left(s_{1}^{2} s_{2} p-z_{1}\right) d x \\
& \eta_{3}=e^{-2 u} s_{1}^{-2}\left(s_{1} d s_{3}+s_{3} d s_{1}+q d q+s_{1}\left(s_{3}+s_{1}^{2} z_{2}\right) d y\right)
\end{aligned}
$$

where $s_{1}=b_{1}^{1}, s_{2}=b_{2}^{1}, s_{3}=b_{1}^{1} b_{2}^{2}, z_{1}$ and $z_{2}$ are arbitrary parameters, with the structure equations

$$
d \theta^{1}=-\theta^{2} \wedge \theta^{3}-\theta^{4} \wedge \theta^{5}
$$




$$
\begin{aligned}
& d \theta^{2}=\eta_{1} \wedge \theta^{2}+\eta_{2} \wedge \omega^{3}-\theta^{1} \wedge \theta^{5}, \\
& d \theta^{3}=-\eta_{1} \wedge \theta^{3}, \\
& d \theta^{4}=-\eta_{1} \wedge \theta^{2}+\eta_{3} \wedge \theta^{5}-\theta^{1} \wedge\left(\theta^{3}+\theta^{4}\right), \\
& d \theta^{5}=\left(\eta_{1}+\theta^{1}\right) \wedge \theta^{5} \\
& d \eta_{1}=-\theta^{3} \wedge \theta^{4}+\theta^{2} \wedge \theta^{3} \\
& d \eta_{2}=\pi_{1} \wedge \theta^{3}+2 \eta_{1} \wedge \eta_{2}, \\
& d \eta_{3}=\pi_{2} \wedge \theta^{5}-2\left(\theta^{1}+\eta_{1}\right) \wedge \eta_{3} .
\end{aligned}
$$

Thus the method is quite simple. It does not require writing out defining systems for symmetry generators. Also, it gives Maurer-Cartan forms, differential invariants, and invariant derivatives for symmetry groups explicitly. But a lot of preliminary work needs to be done before the method becomes applicable to a given differential equation. It is neccessary to find a formulation of the equation in terms of ideals of exterior forms convenient for setting up the equivalence problem for coframes.

\section{Taylor series expansion of defining equations for infinitesimal generators of transitive Lie pseudo-groups}

The second method was provided in [31, 32]. It extracts information about Cartan's structure equations from defining systems for infinitesimal generators. The basic point is that in the case of a transitive symmetry group the explicit form of solutions of the defining system is not required for computing coefficients of the structure equations. These coefficients depend on the finite order Taylor series expansions of the generators, while the information about the Taylor series can be obtained from the involutive form of the defining system.

When the defining system for the infinitesimal generator $X=\sum_{i=1}^{n} \xi^{i} \frac{\partial}{\partial x^{i}}$ of a transitive Lie pseudo-group $\mathcal{G}$ is of the first order, the procedure of the method is as follows. Let $\mathcal{P}_{1}$ be the set of all parametric derivatives of the defining system. Denote

$$
\frac{\partial \xi^{i}}{\partial x^{j}}=\phi^{\rho}, \quad \rho \in\left\{1, \ldots, \#\left(\mathcal{P}_{1}\right)\right\}
$$

for all $\frac{\partial \xi^{i}}{\partial x^{j}} \in \mathcal{P}_{1}$. Then the involutive form of the defining system is

$$
\frac{\partial \xi^{i}}{\partial x^{j}}=\sum_{\rho=1}^{\#\left(\mathcal{P}_{1}\right)} A_{j \rho}^{i}(x) \phi^{\rho}+\sum_{k=1}^{n} b_{j k}^{i}(x) \xi^{k}
$$

for all principal derivaties. As it is shown in [32], in this case Cartan's structure equations of the pseudo-group $\mathcal{G}$ have the form

$$
d \omega^{i}=\sum_{\rho=1}^{\#\left(\mathcal{P}_{1}\right)} \sum_{j=1}^{n} a_{j \rho}^{i} \pi^{\rho} \wedge \omega^{j}+\sum_{1 \leq j<k \leq n} c_{j k}^{i} \omega^{j} \wedge \omega^{k}, \quad i \in\{1, \ldots, n\},
$$

with $a_{j \rho}^{i}=A_{j \rho}^{i}\left(x_{0}\right)$ and $c_{j k}^{i}=b_{k j}^{i}\left(x_{0}\right)-b_{j k}^{i}\left(x_{0}\right)$, where $x_{0}$ is any non-singular point of the defining system (4).

Example 1. (See [31, Example 6].) Consider the Lie pseudo-group on $\mathbb{R}^{2}$ whose infinitesimal generator $X=\xi \partial_{x}+\eta \partial_{y}$ satisfies the defining system

$$
\xi_{x}=\frac{1}{y} \eta, \quad \xi_{y}=0, \quad \eta_{y}=\frac{1}{y} \eta .
$$


In this system, the only parametric derivative of the first order is $\eta_{x}$. We denote it by $\phi^{1}$ and add the only parametric equation (3):

$$
\eta_{x}=\phi^{1} .
$$

Therefore, in any non-singular point $\left(x_{0}, y_{0}\right)$ with $y_{0} \neq 0$ we have $A_{11}^{2}=1, b_{12}^{1}=y_{0}^{-1}, b_{11}^{1}=$ $b_{21}^{1}=b_{22}^{1}=b_{i j}^{2}=0$. This yields the structure equations

$$
d \omega^{1}=-\frac{1}{y_{0}} \omega^{1} \wedge \omega^{2}, \quad d \omega^{2}=\pi^{1} \wedge \omega^{1} .
$$

Any value $y_{0} \neq 0$ is suitable as an initial data point, so we choose $y_{0}=1$.

Additional work should be done when the order of the defining system is greater then $1[32$, Section 4.4].

Example 2. (See [31, Example 8].) For the symmetry group of Liouville's equation (1) the coefficients of the infinitesimal generator $X=\xi \partial_{x}+\eta \partial_{y}+\tau \partial_{u}$ satisfy the defining system in involutive form

$$
\tau_{y y}=-\eta_{y}, \quad \eta_{x y}=0, \quad \xi_{x}=-\tau_{y}-\eta, \quad \xi_{y}=0, \quad \xi_{u}=0, \quad \tau_{x}=0, \quad \tau_{u}=0, \quad \eta_{u}=0 .
$$

The algorithm of [32] gives the following form of the Cartan structure equations:

$$
\begin{aligned}
& d \omega^{1}=-\omega^{1} \wedge \omega^{6}, \\
& d \omega^{2}=-\omega^{2} \wedge \omega^{3}+\omega^{2} \wedge \omega^{6}, \\
& d \omega^{3}=-\omega^{1} \wedge \omega^{4}-\omega^{2} \wedge \omega^{5}, \\
& d \omega^{4}=\pi^{1} \wedge \omega^{1}+\omega^{4} \wedge \omega^{6}, \\
& d \omega^{5}=\pi^{2} \wedge \omega^{2}-\omega^{3} \wedge \omega^{5}-\omega^{5} \wedge \omega^{6}, \\
& d \omega^{6}=-\omega^{1} \wedge \omega^{4} .
\end{aligned}
$$

This result coincides with the previous one: the substitution of $\theta^{1}=\omega_{3}, \theta^{2}=-\omega_{2}-\omega_{4}, \theta^{3}=\omega_{1}$, $\theta^{4}=-\omega_{1}-\omega_{5}, \theta^{5}=\omega_{2}, \eta_{1}=\omega_{6}, \eta_{2}=-\pi_{1}$, and $\eta_{3}=-\pi_{2}$ into (2) gives (6).

The method has the following properties. It is not universal since it is not applicable to differential equations with intransitive symmetry pseudo-groups. Also, it does not give MaurerCartan forms and differential invariants explicitly. An integration should be used to find them from the structure equations [14, Section 7.6].

\section{Invariantized defining equations for Maurer-Cartan forms}

The third method is developed in $[38,39,9]$. It is based on use of invariantized defining equations for Maurer-Cartan forms of Lie pseudo-groups.

Let $\mathcal{D}(M)$ be the pseudo-group of local diffeomorphisms $Z=\phi(z)$ of a manifold $M, \operatorname{dim} M=m$, and $\mathcal{D}^{(\infty)}(M)$ be the bundle of $\infty$-jets of maps in $\mathcal{D}(M)$. Local coordinates of the base space $M$ and $\mathcal{D}^{(\infty)}(M)$ are denoted by $z=\left(z^{i}\right)$ and $\left(z^{i}, Z_{I}^{\alpha}\right)$, respectively, where $Z=\left(Z^{\alpha}\right)$ are target coordinates for $\phi \in \mathcal{D}(M), \phi(z)=Z$, and $Z_{I}^{\alpha}=\partial^{I} Z^{\alpha} / \partial z^{I}=\partial^{i_{1}+\cdots+i_{m}} Z^{\alpha} /\left(\partial z^{1}\right)^{i_{1}} \cdots\left(\partial z^{m}\right)^{i_{m}}$ for $I=\left(i_{1}, i_{2}, \ldots, i_{m}\right)$ (our notation for multi-indexes differs slightly from those of $[38,39,9]$ ). Then, as it is shown in [38], the Maurer-Cartan forms for $\mathcal{D}^{(\infty)}(M)$ are $\sigma^{\alpha}=Z_{j}^{\alpha} d z^{j}, \mu^{\alpha}=d Z^{\alpha}-$ $Z_{j}^{\alpha} d z^{j}$, and $\mu_{I}^{\alpha}=\mathbb{D}_{Z}^{I}\left(\mu^{\alpha}\right), \alpha \in\{1, \ldots, m\}, \# I=i_{1}+\cdots+i_{m} \geq 1$, where $\mathbb{D}_{Z}^{I}=\mathbb{D}_{Z^{1}}^{i_{1}} \circ \cdots \circ \mathbb{D}_{Z^{m}}^{i_{m}}$,

$$
\mathbb{D}_{Z^{j}}=\sum_{i=1}^{m} w_{j}^{i} \mathbb{D}_{z^{i}}, \quad \mathbb{D}_{z^{i}}=\frac{\partial}{\partial z^{i}}+\sum_{\alpha=1}^{m} \sum_{\# I \geq 0} Z_{I+1_{i}}^{\alpha} \frac{\partial}{\partial Z_{I}^{\alpha}},
$$


while $\left(w_{j}^{i}\right)$ is the inverse matrix for the Jacobian matrix $\left(Z_{j}^{\alpha}\right)$. The structure equations for $\mathcal{D}^{(\infty)}(M)$ have the form

$$
\begin{aligned}
& d \mu \llbracket H \rrbracket=\nabla \mu \llbracket H \rrbracket \wedge(\mu \llbracket H \rrbracket-d Z), \\
& d \sigma=-d \mu \llbracket 0 \rrbracket,
\end{aligned}
$$

where $\mu \llbracket H \rrbracket=\left(\mu^{\alpha} \llbracket H \rrbracket\right)=\left(\sum_{\# I \geq 0} \frac{1}{I !} \mu_{I}^{\alpha} H^{I}\right), H^{I}=H_{1}^{i_{1}} \cdots H_{m}^{i_{m}}$, and $\nabla \mu \llbracket H \rrbracket=\left(\frac{\partial \mu^{\alpha} \llbracket H \rrbracket}{\partial H_{j}}\right)$ denote the Jacobian matrix of the vector $\mu \llbracket H \rrbracket$ of power series in the variables $H=\left(H_{j}\right)$.

Let $V=\zeta^{\alpha} \partial_{z^{\alpha}}$ be the infinitesimal generators of a sub-pseudo-group $\mathcal{G} \subset \mathcal{D}(M)$. They are characterized by the defining equations

$$
L\left(z^{i}, \zeta_{I}^{\alpha}\right)=0 .
$$

If $\mathcal{G}$ is the symmetry group of a system of differential equations, then (9) are (the involutive completion of) the usual determining equations obtained through Lie's infinitesimal technique.

The method is based on the following theorems [38]:

Theorem 1. The invariant forms $\mu_{I}^{\alpha}$ of a Lie pseudo-group $\mathcal{G} \subset \mathcal{D}(M)$ satisfy the linear system

$$
L\left(Z^{i}, \mu_{I}^{\alpha}\right)=0,
$$

obtained by replacing $z^{i}$ by $Z^{i}$ and $\zeta_{I}^{\alpha}$ by $\mu_{I}^{\alpha}$ in the determining equations (9).

Theorem 2. The structure equations of the invariant coframe for a Lie pseudo-group $\mathcal{G}$ are obtained by restricting the diffeomorphism structure equations (7), (8) to the space of solutions of the equations (10).

These results allow to find the structure equations of the symmetry group of a system of differential equations from an involutive form of infinitesimal defining equations. The required computations rely exclusively on linear algebra and differentiation, and can be readily implemented in any standard symbolic computation package.

Example 3. To illustrate the method, we apply it to Liouville's equation (1). We denote $\left(z^{1}, z^{2}, z^{3}\right)=(x, y, u),\left(Z^{1}, Z^{2}, Z^{3}\right)=(X, Y, U)$, and take the infinitesimal defining system in the involutive form (5). Then from the fourth and fifth equations of (5) and their derivatives w.r.t. $y$ and $u$ we have $\mu_{(0, k, l)}^{1}=0$ for $k+l \geq 1$, so

$$
\mu^{1} \llbracket H \rrbracket=\sum_{j \geq 0} \frac{1}{j !} \mu_{(j, 0,0)}^{1} H_{1}^{j} .
$$

Further, from the sixth and seventh equations of (5) and their derivatives w.r.t. $x$ and $u$ we have $\mu_{(j, 0, l)}^{2}=0$ for $j+l \geq 1$, thus

$$
\mu^{2} \llbracket H \rrbracket=\sum_{k \geq 0} \frac{1}{k !} \mu_{(0, k, 0)}^{2} H_{2}^{k} .
$$

Finally, from the third equation of (5) and its derivatives w.r.t. $x, y$, and $u$ we have $\mu_{(0,0,0)}^{3}=$ $-\mu_{(1,0,0)}^{1}-\mu_{(0,1,0)}^{2}$ and

$$
\mu^{3} \llbracket H \rrbracket=-\sum_{j \geq 0} \frac{1}{j !} \mu_{(j+1,0,0)}^{1} H_{1}^{j}-\sum_{k \geq 0} \frac{1}{k !} \mu_{(0, k+1,0)}^{2} H_{2}^{k} .
$$


Let us denote $\mu_{(j, 0,0)}^{1}=\phi_{j}$ and $\mu_{(0, k, 0)}^{2}=\psi_{k}$ for brevity. Then substitution of $\mu^{1} \llbracket H \rrbracket, \mu^{2} \llbracket H \rrbracket$, and $\mu^{3} \llbracket H \rrbracket$ into (7) and (8) yields an infinite system of structure equations:

$$
\begin{aligned}
& \left(\begin{array}{c}
\sum_{j \geq 0} \frac{1}{j !} d \phi_{j} H_{1}^{j} \\
\sum_{k \geq 0} \frac{1}{k !} d \psi_{k} H_{2}^{k} \\
-\sum_{j \geq 0} \frac{1}{j !} d \phi_{j+1} H_{1}^{j}-\sum_{k \geq 0} \frac{1}{k !} d \psi_{k+1} H_{2}^{k}
\end{array}\right) \\
& =\left(\begin{array}{ccc}
\sum_{j \geq 0} \frac{1}{j !} \phi_{j+1} H_{1}^{j} & 0 & 0 \\
0 & \sum_{k \geq 0} \frac{1}{k !} \psi_{k+1} H_{2}^{k} & 0 \\
-\sum_{j \geq 0} \frac{1}{j !} \phi_{j+2} H_{1}^{j} & \sum_{k \geq 0} \frac{1}{k !} \psi_{k+2} H_{2}^{k} & 0
\end{array}\right) \wedge\left(\begin{array}{c}
-\sigma^{1}+\sum_{j \geq 1} \frac{1}{j !} \phi_{j} H_{1}^{j} \\
-\sigma^{2}+\sum_{k \geq 1} \frac{1}{k !} \psi_{k} H_{2}^{k} \\
-\sigma^{3}-\sum_{j \geq 1} \frac{1}{j !} \phi_{j+1} H_{1}^{j}-\sum_{k \geq 1} \frac{1}{k !} \psi_{k+1} H_{2}^{k}
\end{array}\right)
\end{aligned}
$$

and

$$
d \sigma^{1}=-d \phi_{0}, \quad d \sigma^{2}=-d \psi_{0}, \quad d \sigma^{3}=d \phi_{1}+d \psi_{1} .
$$

From these equations we have

$$
\begin{aligned}
& d \phi_{0}=-\phi_{1} \wedge \sigma^{1}, \\
& d \phi_{j}=-\phi_{j+1} \wedge \sigma^{1}+\sum_{p \geq 0, q \geq 1, p+q=j} \frac{j !}{p ! q !} \phi_{p+1} \wedge \phi_{q}, \quad j \geq 1, \\
& d \psi_{0}=-\psi_{1} \wedge \sigma^{2}, \\
& d \psi_{k}=-\phi_{k+1} \wedge \sigma^{2}+\sum_{p \geq 0, q \geq 1, p+q=k} \frac{k !}{p ! q !} \psi_{p+1} \wedge \psi_{q}, \quad k \geq 1, \\
& d \sigma^{1}=\phi_{1} \wedge \sigma^{1}, \\
& d \sigma^{2}=\psi_{1} \wedge \sigma^{2}, \\
& d \sigma^{3}=-\phi_{2} \wedge \sigma^{1}-\psi_{2} \wedge \sigma^{2} .
\end{aligned}
$$

To establish correspondence with the previous results, we note that the substitution of $\sigma^{1}=$ $\omega^{1}, \sigma^{2}=\omega^{2}, \phi_{1}=\omega^{6}, \phi_{2}=\omega^{4}, \psi_{1}=\omega^{3}-\omega^{6}, \psi_{2}=\omega^{5}, \phi_{3}=-\pi_{1}$, and $\psi_{3}=-\pi_{2}$ into equations (11) for $d \sigma_{1}, d \sigma_{2}, d \phi_{1}, d \phi_{2}, d \psi_{1}$, and $d \psi_{2}$ gives equations (6), while equations (11) for $d \phi_{i+1}$ and $d \psi_{k+1}$ appear from the exterior differentials of the equations for $d \phi_{i}$ and $d \psi_{k}$, respectively. Therefore, equations (11) are infinite prolongation of equations (6).

The sets of equations (11) for $\sigma^{1}, \phi_{i}$, and $\sigma^{2}, \psi_{k}$ are independent. Moreover, each of these sets coincides with the structure equations for the infinite prolongation of the diffeomorphism pseudogroup on $\mathbb{R}^{1}$ [5], [38, Example 4.1]. This shows that the pseudo-group of contact symmetries of Liouville's equation is a direct product of two diffeomorphism pseudo-groups on $\mathbb{R}^{1}$, as it follows, of course, from the results obtained by the infinitesimal method [30, Bd. 5, 469-478].

Unlike the previous two methods, the method of $[38,39,9]$ is universal since it is applicable to any differential equation. It requires analysis of the defining systems for infinitesimal generators and its reduction to the involutive form. For differential equations with infinite symmetry pseudo-groups the method produces infinite sets of Maurer-Cartan forms and infinite systems of structure equations. Also, the Maurer-Cartan forms obtained by this method depend on both source and target variables of the diffeomorphism pseudo-group. Therefore, additional work needs to be done to obtain finite sets of Maurer-Cartan forms and to express them in terms which are suitable for further implementations such as finding transformations between equivalent equations. 


\section{The moving coframe method}

Finally, the fourth approach is based on the moving coframe method provided in [12]. Applied to contact symmetries of differential equations of the second order, the method has the following outline. Consider the bundle $J^{2}(\varepsilon)$ of the second order jets of local sections of the trivial bundle $\mathcal{E}=\mathbb{R}^{n} \times \mathbb{R} \rightarrow \mathbb{R}^{n}$. Contact transformations $\Delta: J^{2}(\mathcal{E}) \rightarrow J^{2}(\mathcal{E}), \Delta:\left(x^{i}, u, p_{i}, p_{i j}\right) \mapsto$ $\left(\bar{x}^{i}, \bar{u}, \bar{p}_{i}, \bar{p}_{i j}\right)$, are characterized by requirements $\Delta^{*}\left(d \bar{u}-\bar{p}_{i} d \bar{x}^{i}\right) \equiv 0\left(\bmod d u-p_{i} d x^{i}\right)$ and $\Delta^{*}\left(d \bar{p}_{i}-\bar{p}_{i j} d \bar{x}^{j}\right) \equiv 0\left(\bmod d u-p_{i} d x^{i}, d p_{i}-p_{i j} d x^{j}\right)$. Therefore, Maurer-Cartan forms for the pseudo-group Cont $\left(J^{2}(\varepsilon)\right)$ of contact transformations on $J^{2}(\varepsilon)$ can be easily found using Cartan's equivalence method, see, e.g., [35]. These forms are

$$
\begin{aligned}
& \Theta_{0}=a\left(d u-p_{i} d x^{i}\right), \\
& \Theta_{i}=g_{i} \Theta_{0}+a B_{i}^{k}\left(d p_{k}-p_{k l} d x^{l}\right), \\
& \Xi^{i}=c^{i} \Theta_{0}+f^{i k} \Theta_{k}+b_{k}^{i} d x^{k}, \\
& \Sigma_{i j}=s_{i j} \Theta_{0}+w_{i j}^{k} \Theta_{k}+z_{i j k} \Xi^{k}+a B_{i}^{k} B_{j}^{l} d p_{k l},
\end{aligned}
$$

where $i, j \in\{1, \ldots, n\}, a \neq 0$, det $\left(b_{j}^{i}\right) \neq 0, f^{i k}=f^{k i}, s_{i j}=s_{j i}, w_{i j}^{k}=w_{j i}^{k}, z_{i j k}=z_{j i k}=z_{i k j}$, while $\left(B_{j}^{i}\right)$ is the inverse matrix for the matrix $\left(b_{j}^{i}\right)$. The structure equations of $\operatorname{Cont}\left(J^{2}(\mathcal{E})\right)$ have the following form:

$$
\begin{aligned}
& d \Theta_{0}=\Phi_{0}^{0} \wedge \Theta_{0}+\Xi^{i} \wedge \Theta_{i}, \\
& d \Theta_{i}=\Phi_{i}^{0} \wedge \Theta_{0}+\Phi_{i}^{k} \wedge \Theta_{k}+\Xi^{k} \wedge \Sigma_{i k}, \\
& d \Xi^{i}=\Phi_{0}^{0} \wedge \Xi^{i}-\Phi_{k}^{i} \wedge \Xi^{k}+\Psi^{i 0} \wedge \Theta_{0}+\Psi^{i k} \wedge \Theta_{k}, \\
& d \Sigma_{i j}=\Phi_{i}^{k} \wedge \Sigma_{k j}-\Phi_{0}^{0} \wedge \Sigma_{i j}+\Upsilon_{i j}^{0} \wedge \Theta_{0}+\Upsilon_{i j}^{k} \wedge \Theta_{k}+\Lambda_{i j k} \wedge \Xi^{k} .
\end{aligned}
$$

A differential equation $\mathcal{R}$ of the second order is a subbundle of $J^{2}(\mathcal{E})$. Let $\iota: \mathcal{R} \rightarrow J^{2}(\mathcal{E})$ be the inclusion map. Then we can find the Maurer-Cartan forms for the pseudo-group $\operatorname{Sym}(\mathcal{R})$ of contact symmetries of $\mathcal{R}$ from the restrictions $\theta_{0}=\iota^{*} \Theta_{0}, \theta_{i}=\iota^{*} \Theta_{i}, \xi^{i}=\iota^{*} \Xi^{i}, \sigma_{i j}=\iota^{*} \Sigma_{i j}$ by standard procedures of Cartan's equivalence method, see $[12,33,34]$ for details.

Example 4. Applying the moving coframe method to the symmetry pseudo-group of Liouville's equation (1), we obtain the Maurer-Cartan forms

$$
\begin{aligned}
& \theta_{0}=d u-u_{x} d x-u_{y} d y \\
& \theta_{1}=r_{1}^{-1}\left(d u_{x}-u_{x x} d x-e^{u} d y\right) \\
& \theta_{2}=r_{1} e^{-u}\left(d u_{y}-e^{u} d x-u_{y y} d y\right) \\
& \xi^{1}=r_{1} d x \\
& \xi^{2}=r_{1}^{-1} e^{u} d y \\
& \sigma_{11}=r_{1}^{-2}\left(d u_{x x}-u_{x} d u_{x}+\left(u_{x} u_{x x}+r_{1}^{3} r_{2}\right) d x\right) \\
& \sigma_{22}=r_{1}^{2} e^{-2 u}\left(d u_{y y}-u_{y} d u_{y}+\left(u_{y} u_{y y}+r_{1}^{-3} r_{3}\right) d y\right) \\
& \eta_{1}=r_{1}^{-1}\left(d r_{1}-u_{x} \xi^{1}\right) \\
& \eta_{2}=d r_{2}-3 r_{2} \eta_{1}+r_{1}^{-2}\left(u_{x x}+u_{x}^{2}\right)\left(\theta_{1}+\xi^{2}\right)+3 r_{1}^{-1} u_{x} \sigma_{11}+v_{1} \xi^{1}, \\
& \eta_{3}=d r_{3}+3 r_{3}\left(\eta_{1}+\theta_{0}\right)+r_{1}^{2} e^{-2 u}\left(u_{y y}+u_{y}^{2}\right)\left(\theta_{2}+\xi^{1}\right)+3 r_{1} e^{-u} u_{y} \sigma_{22}+v_{2} \xi^{2},
\end{aligned}
$$

where $r_{1}=b_{1}^{1}, r_{2}=z_{111}, r_{3}=z_{222}, v_{1}$ and $v_{2}$ are arbitrary parameters, while $\sigma_{12}=0$. The forms satisfy the following structure equations:

$$
d \theta_{0}=-\theta_{1} \wedge \xi^{1}-\theta_{2} \wedge \xi^{2},
$$




$$
\begin{aligned}
& d \theta_{1}=\eta_{1} \wedge \theta_{1}-\theta_{0} \wedge \xi^{2}+\xi^{1} \wedge \sigma_{11}, \\
& d \theta_{2}=-\eta_{1} \wedge \theta_{2}-\theta_{0} \wedge\left(\theta_{2}+\xi^{1}\right)+\xi^{2} \wedge \sigma_{22}, \\
& d \xi^{1}=-\eta_{1} \wedge \xi^{1} \\
& d \xi^{2}=\left(\eta_{1}+\theta_{0}\right) \wedge \xi^{2}, \\
& d \sigma_{11}=\eta_{2} \wedge \xi^{1}+2 \eta_{1} \wedge \sigma_{11}, \\
& d \sigma_{22}=\eta_{3} \wedge \xi^{2}-2\left(\eta_{1}+\theta_{0}\right) \wedge \sigma_{22}, \\
& d \eta_{1}=\left(\theta_{1}+\xi^{2}\right) \wedge \xi^{1}, \\
& d \eta_{2}=\pi_{1} \wedge \xi^{1}+3 \eta_{1} \wedge \eta_{2}+2\left(\theta_{1}+\xi^{2}\right) \wedge \sigma_{11}, \\
& d \eta_{3}=\pi_{2} \wedge \xi^{2}-3\left(\eta_{1}+\theta_{0}\right) \wedge \eta_{3}+2\left(\theta_{2}+\xi^{1}\right) \wedge \sigma_{22} .
\end{aligned}
$$

The substitution of $\xi^{1}=\omega^{1}, \xi^{2}=\omega^{2}, \theta_{0}=\omega^{3}, \theta_{1}=-\omega^{2}-\omega^{4}, \theta_{2}=-\omega^{1}-\omega^{5}, \eta_{1}=-\omega^{6}$, $\eta_{2}=\pi_{1}$, and $\eta_{3}=\pi_{2}$ into (12) yields equations (6). Therefore, this result coincides with the results obtained in previous examples.

The moving coframe method enables one to find Maurer-Cartan forms, differential invariants, and operators of invariant differentiations for symmetry pseudo-groups explicitly, in contrast to the method of [32]. It does not require integration at all, and, unlike the methods of $[32,38,39,9]$, does not use infinitesimal defining systems. Also, the method is universal since it is applicable to any differential equation. The price of these advantages is lengthy and intricate computations. Nevertheless the method allows one to solve effectively problems concerned with symmetry classification and equivalence of differential equation. We illustrate it in the two following sections.

\section{Contact equivalence problem for linear hyperbolic equations}

In [35] the moving coframe method is used to solve the local equivalence problem for the class of linear second order hyperbolic equations in two independent variables

$$
u_{t x}=T(t, x) u_{t}+X(t, x) u_{x}+U(t, x) u
$$

w.r.t. the pseudo-group of contact transformations.

This class has been studied for more than two centuries. In [29] P.S. Laplace found semiinvariants $H=-T_{t}+T X+U$ and $K=-X_{x}+T X+U$. These functions are invariants of the sub-pseudo-group $u \mapsto \lambda(t, x) u, \lambda \neq 0$. Laplace proved that equation (13) is equivalent to the wave equation $u_{t x}=0$ w.r.t. this sub-pseudo-group whenever $H \equiv 0$ and $K \equiv 0$.

S. Lie studied equations (13) by means of the infinitesimal method [30, Bd. 3, 492-523]. He found canonical forms for equations (13) and methods of their integration.

L.V. Ovsiannikov found contact invariants $P=K / H, Q=(\ln |H|)_{t x} / H$, and applied them to the problem of classification of equations (13) with non-trivial finite-dimensional subgroups of symmetry pseudo-groups [41], [42, Section 9.2].

The solution of the contact equivalence problem for the class (13) is found in [35]:

Theorem 3. Class (13) is divided into the six subclasses $\mathfrak{C}_{1}, \mathrm{C}_{2}, \ldots, \mathrm{C}_{6}$ invariant under an action of the pseudo-group of contact transformations:

$\mathcal{C}_{1}$ consists of all equations (13) such that $H \equiv 0$ and $K \equiv 0$;

$\mathrm{C}_{2}$ consists of all equations (13) such that $P_{t} \neq 0$;

$\mathrm{C}_{3}$ consists of all equations (13) such that $P_{t} \equiv 0$ and $P_{x} \neq 0$;

$\mathrm{C}_{4}$ consists of all equations (13) such that $P \equiv$ const and $Q_{t} \neq 0$; 
$\mathrm{C}_{5}$ consists of all equations $(13)$ such that $P \equiv$ const, $Q_{t} \equiv 0$, and $Q_{x} \neq 0$;

$\mathcal{C}_{6}$ consists of all equations (13) such that $P \equiv$ const and $Q \equiv$ const.

Every equation from the subclass $\mathcal{C}_{1}$ is equivalent to the linear wave equation $u_{t x}=0$.

Every equation from the subclass $\mathrm{C}_{6}$ is equivalent to the equation

$$
u_{t x}=-t u_{t}-P x u_{x}-P t x u
$$

when $Q=0$, or to the Euler-Poisson equation

$$
u_{t x}=\frac{2}{Q(t+x)} u_{t}+\frac{2 P}{Q(t+x)} u_{x}-\frac{4 P}{Q^{2}(t+x)^{2}} u
$$

when $Q \neq 0$.

For the subclass $\mathcal{C}_{2}$, the basic invariants are $P, Q$, and $J=\left(H_{t} P_{t}-H P_{t t}\right) H^{-1} P_{t}^{-2}$, the operators of invariant differentiation are $\mathbb{D}_{1}=P_{t}^{-1} D_{t}$ and $\mathbb{D}_{2}=P_{t} H^{-1} D_{x}$.

For $\mathcal{C}_{3}$, the basic invariants are $P, Q$, and $L=\left(H_{x} P_{x}-H P_{x x}\right) H^{-1} P_{x}^{-2}$, the operators of invariant differentiation are $\mathbb{D}_{1}=P_{x} H^{-1} D_{t}$ and $\mathbb{D}_{2}=P_{x}^{-1} D_{x}$.

For $\mathcal{C}_{4}$, the basic invariants are $Q, M_{1}=Q_{t x} H^{-1}$, and $M_{2}=\left(H_{t} Q_{t}-H Q_{t t}\right) H^{-1} Q_{t}^{-2}$, the operators of invariant differentiation are $\mathbb{D}_{1}=Q_{t}^{-1} D_{t}$ and $\mathbb{D}_{2}=Q_{t} H^{-1} D_{x}$.

For $\mathrm{C}_{5}$, the basic invariants are $Q$ and $N=\left(H_{x} Q_{x}-H Q_{x x}\right) H^{-1} Q_{x}^{-2}$, the operators of invariant differentiation are $\mathbb{D}_{1}=Q_{x} H^{-1} D_{t}$ and $\mathbb{D}_{2}=Q_{x}^{-1} D_{x}$.

Two equations from the subclasses $\mathcal{C}_{2}-\mathrm{C}_{5}$ are locally equivalent to each other if and only if they have the same functional dependencies among the basic invariants and their invariant derivatives up to the second order.

Different results were stated in [23] and [22]:

Theorem 4 ([23]). The functions $P, Q$,

$$
\begin{aligned}
& J_{3}^{1}=H^{-3}\left(K H_{t x}+H K_{t x}-H_{t} K_{x}-H_{x} K_{t}\right), \\
& J_{3}^{2}=H^{-9}\left(H K_{x}-K H_{x}\right)^{2}\left(H K H_{t t}-H^{2} K_{t t}-3 K H_{t}^{2}+3 H H_{t} K_{t}\right),
\end{aligned}
$$

and

$$
J_{3}^{3}=H^{-9}\left(H K_{t}-K H_{t}\right)^{2}\left(H K H_{x x}-H^{2} K_{x x}-3 K H_{x}^{2}+3 H H_{x} K_{x}\right)
$$

are a basis of the complete set of invariants of (13). Any other differential invariant is a function of these basic invariants and their invariant derivatives.

Theorem 5 ([22]). A basis of invariants for equations (13) consists of the invariants

$$
P, \quad Q, \quad I=P_{t} P_{x} H^{-1}, \quad \widetilde{Q}=(\ln |K|)_{t x} K^{-1},
$$

or of invariants of the alternative basis

$$
P, \quad Q, \quad I, \quad J=\left(H_{t} P_{t}-H P_{t t}\right) H^{-1} P_{t}^{-2} .
$$

The operators of invariant differentiations are taken in [22] in the following form: $\mathcal{D}_{1}=P_{t}^{-1} D_{t}$ and $\mathcal{D}_{2}=P_{x}^{-1} D_{x}$.

The functions of Theorems 4, 5 do not provide bases of invariants for the whole class of equations (13). To prove this, we consider the following equation:

$$
u_{t x}=u_{t}+\frac{2(p(t)-1)}{q(t)(t+x)} u_{x}+\frac{2(1-(p(t)-1)(t+x))}{q(t)(t+x)^{2}} u
$$


with $p(t) \neq 0, q(t) \neq 0, p^{\prime}(t) \neq 0$, and $q^{\prime}(t) \neq 0$. For this equation we have $P=p(t), Q=q(t)$, $J_{3}^{1}=2 p(t) q(t), J_{3}^{2}=J_{3}^{3}=I=0, \widetilde{Q}=q(t)(p(t))^{-1}$, while

$$
J=-\frac{2}{q^{\prime}(t)(t+x)}-\frac{p^{\prime \prime}(t)}{\left(p^{\prime}(t)\right)^{2}}-\frac{q^{\prime}(t)}{p^{\prime}(t) q(t)} .
$$

Since $J$ depends on $x$ explicitly, it is functionally independent of $P, Q, J_{3}^{1}, J_{3}^{2}, J_{3}^{3}, I$, and $\widetilde{Q}$. Therefore, the functions from Theorem 4 and the first set of functions from Theorem 5 do not provide a basis of differential invariants for the whole class (13).

Otherwise, for Moutard's equation

$$
u_{t x}=U(t, x) u
$$

we have $\widetilde{Q}=Q, P=1$, so $P_{t}=P_{x}=I=0$. Therefore the invariant $J$ and the operators $\mathcal{D}_{1}=P_{t}^{-1} D_{t}, \mathcal{D}_{2}=P_{x}^{-1} D_{x}$ are not defined for this equation. This proves that the both sets of functions from Theorem 5 do not provide bases of invariants for the subclass (16).

Remark 1. As it is shown in [35], the symmetry pseudo-groups of equations (13) from the subclasses $\mathcal{C}_{2}-\mathcal{C}_{5}$ are intransitive. Therefore, the moving coframe method is shown to be applicable to study intransitive symmetry pseudo-groups as well.

\section{Linearizability of the generalized Hunter-Saxton equation}

In this section, we use the moving coframe method to study the generalized Hunter-Saxton equation

$$
u_{t x}=u u_{x x}+\kappa u_{x}^{2}
$$

This equation has a number of applications in the nonlinear instability theory of a director field of a liquid crystal [20], in geometry of Einstein-Weil spaces [46, 18], in constructing partially invariant solutions for the Euler equations of an ideal fluid [16], and has been a subject of many recent investigations. In the case $\kappa=\frac{1}{2}$ the general solution [20], the tri-Hamiltonian formulation [40], the pseudo-spherical formulation and the quadratic pseudo-potentials [44] have been found. The conjecture of linearizability of equation (17) in the case $\kappa=-1$ has been made in [16]. In [43] a formula for the general solution of (17) has been proposed. This formula uses a nonlocal change of variables.

We prove that equation (17) is equivalent under a contact transformation to the Euler-Poisson equation (14) with $P=2(1-\kappa)$ and $Q=2 \kappa$ :

$$
u_{t x}=\frac{1}{\kappa(t+x)} u_{t}+\frac{2(1-\kappa)}{\kappa(t+x)} u_{x}-\frac{2(1-\kappa)}{(\kappa(t+x))^{2}} u .
$$

Also, we find the general solution of (17) in terms of local variables.

Using the moving coframe method, we obtain the structure equations for the symmetry pseudo-group of equation (18) in the form

$$
\begin{aligned}
& d \theta_{0}=\eta_{1} \wedge \theta_{0}+\xi^{1} \wedge \theta_{1}+\xi^{2} \wedge \theta_{2}, \\
& d \theta_{1}=\eta_{2} \wedge \theta_{1}-2(1-\kappa) \theta_{0} \wedge \xi^{2}+\xi^{1} \wedge \sigma_{11}, \\
& d \theta_{2}=\left(2 \eta_{1}-\eta_{2}\right) \wedge \theta_{2}-\theta_{0} \wedge \xi^{1}+\xi^{2} \wedge \sigma_{22}, \\
& d \xi^{1}=\left(\eta_{1}-\eta_{2}\right) \wedge \xi^{1}, \\
& d \xi^{2}=\left(\eta_{2}-\eta_{1}\right) \wedge \xi^{2},
\end{aligned}
$$




$$
\begin{aligned}
& d \sigma_{11}=\left(2 \eta_{2}-\eta_{1}\right) \wedge \sigma_{11}+\eta_{3} \wedge \xi^{1}+3(2 \kappa-1) \theta_{1} \wedge \xi^{2}, \\
& d \sigma_{22}=\left(3 \eta_{1}-2 \eta_{2}\right) \wedge \sigma_{22}+\eta_{4} \wedge \xi^{2} \\
& d \eta_{1}=(2 \kappa-1) \xi^{1} \wedge \xi^{2} \\
& d \eta_{2}=(1-4 \kappa) \xi^{1} \wedge \xi^{2}, \\
& d \eta_{3}=\pi_{1} \wedge \xi^{1}-\left(2 \eta_{1}-3 \eta_{2}\right) \wedge \eta_{3}+4(3 \kappa-1) \xi^{2} \wedge \sigma_{11}, \\
& d \eta_{4}=\pi_{2} \wedge \xi^{2}+\left(4 \eta_{1}-3 \eta_{2}\right) \wedge \eta_{4}+2(3-\kappa) \xi^{1} \wedge \sigma_{22},
\end{aligned}
$$

with $\theta_{0}=a\left(d u-u_{t} d t-u_{x} d x\right), \theta_{1}=a b^{-1}\left(d u_{t}-u_{t t} d t-R_{2} d x\right)+2(\kappa-1)(\kappa b(t+x))^{-1} \theta_{0}, \theta_{2}=$ $a b \kappa(t+x)^{2}\left(d u_{x}-R_{2} d t-u_{x x} d x\right)+b(t+x) \theta_{0}, \xi^{1}=b d t$, and $\xi^{2}=b^{-1} \kappa^{-1}(t+x)^{-2} d x$, where $R_{2}$ is the right-hand side of equation (18), while $a$ and $b$ are arbitrary non-zero constants. The forms $\sigma_{11}, \ldots, \pi_{2}$ are too long to be written out in full here. We write equation (17) and its MaurerCartan forms in tilded variables, then similar computations give $\widetilde{\theta}_{0}=\widetilde{a}\left(d \widetilde{u}-\widetilde{u}_{\widetilde{t}} d \widetilde{t}-\widetilde{u}_{\widetilde{x}} d \widetilde{x}\right), \widetilde{\theta}_{1}=$ $\widetilde{a} \widetilde{b}^{-1}\left(d \widetilde{u}_{\tilde{t}}-\widetilde{u}_{\widetilde{t t}} d \widetilde{t}-\widetilde{R}_{1} d \widetilde{x}\right)-\widetilde{b}^{-2} \widetilde{u} \widetilde{u}_{\widetilde{x} \widetilde{x}} \widetilde{\theta}_{2}-(2 \kappa-1) \widetilde{b} \widetilde{u}_{\widetilde{x}} \widetilde{\theta}_{0}, \widetilde{\theta}_{2}=\widetilde{a} \widetilde{b}^{-1}\left(\widetilde{u}_{\widetilde{x} \widetilde{x}}\right)^{-1}\left(d \widetilde{u}_{\widetilde{x}}-\widetilde{R}_{1} d \widetilde{t}-\widetilde{u}_{\widetilde{x} \widetilde{x}} d \widetilde{x}\right)$, $\widetilde{\xi}^{1}=\widetilde{b} d \widetilde{t}$, and $\widetilde{\xi}^{2}=\widetilde{b}^{-1}\left(d \widetilde{u}_{\widetilde{x}}-\kappa\left(\widetilde{u}_{\widetilde{x}}\right)^{2} d \widetilde{t}\right)$, where $\widetilde{R}_{1}$ is the right-hand side of equation (17) written in the tilded vatiables, while $\widetilde{a}$ and $\widetilde{b}$ are arbitrary non-zero constants. The forms $\widetilde{\sigma}_{11}, \ldots, \widetilde{\pi}_{2}$ are too long to be written out in full. The structure equations for (17) differ from (19) only in replacing $\theta_{0}, \ldots, \pi_{2}$ by their tilded counterparts. Therefore, results of Cartan's method (see, e.g., [37, Theorem 15.12]) yield the contact equivalence of equations (17) and (18). Since the Maurer-Cartan forms for both symmetry groups are known, the equivalence transformation $\Psi:\left(t, x, u, u_{t}, u_{x}\right) \mapsto\left(\widetilde{t}, \widetilde{x}, \widetilde{u}, \widetilde{u}_{\tilde{t}}, \widetilde{u}_{\widetilde{x}}\right)$ can be found from the requirements $\Psi^{*} \widetilde{\theta}_{0}=\theta_{0}, \Psi^{*} \widetilde{\theta}_{1}=\theta_{1}$, $\Psi^{*} \widetilde{\theta}_{2}=\theta_{2}, \Psi^{*} \widetilde{\xi}^{1}=\xi^{1}$, and $\Psi^{*} \widetilde{\xi}^{2}=\xi^{2}$ :

Theorem 6. The contact transformation $\Psi$

$$
\begin{aligned}
& \widetilde{u}=(t+x)^{-\frac{1}{\kappa}}\left(\kappa(t+x) u_{x}+(\kappa-1) u\right), \\
& \widetilde{t}=\kappa^{-1} t, \\
& \widetilde{x}=-(t+x)^{\frac{\kappa-1}{\kappa}}\left(\kappa(t+x) u_{x}-u\right), \\
& \widetilde{u}_{\widetilde{t}}=\kappa^{2}(t+x)^{-\frac{1}{\kappa}}\left(u_{t}-u_{x}\right), \\
& \widetilde{u}_{\widetilde{x}}=-(t+x)^{-1}
\end{aligned}
$$

takes the Euler-Poisson equation (18) to the generalized Hunter-Saxton equation (17) (written in the tilded variables).

Equation (18) has an intermediate integral, and its general solution can be found in quadratures. Indeed, for the invariants of this equation we have $P+Q=2$, therefore the Laplace $t$-transformation, [42, Section 9.3], takes equation (18) to a factorizable linear hyperbolic equation. Namely, we consider the system

$$
\begin{aligned}
& v=u_{x}-(\kappa(t+x))^{-1} u, \\
& v_{t}=2(1-\kappa)(\kappa(t+x))^{-1} v+\kappa^{-1}(t+x)^{-2} u .
\end{aligned}
$$

Substituting (20) into (21) yields equation (18), while expressing $u$ from (21) and substituting it into (20) gives the equation

$$
v_{t x}=\frac{1-2 \kappa}{\kappa(t+x)} v_{t}+\frac{2(\kappa-1)}{\kappa(t+x)} v_{x}-\frac{(2 \kappa-1)(\kappa-2)}{(\kappa(t+x))^{2}} v
$$

with the trivial Laplace semi-invariant $H$. Hence, the substitution

$$
w=v_{x}+(2 \kappa-1)(\kappa(t+x))^{-1} v
$$


takes equation (22) into the equation

$$
w_{t}=-2(\kappa-1)(\kappa(t+x))^{-1} w .
$$

Integrating (24) and (23), we have the general solution for equation (22):

$$
v=(t+x)^{\frac{1-2 \kappa}{\kappa}}\left(S(t)+\int R(x)(t+x)^{\frac{1}{\kappa}} d x\right),
$$

where $S(t)$ and $R(x)$ are arbitrary smooth functions of their arguments. Then equation (21) gives the general solution for equation (18):

$$
u=(t+x)^{\frac{1}{\kappa}}\left(\kappa S^{\prime}(t)+\int R(x)(t+x)^{\frac{1-\kappa}{\kappa}} d x\right)-(t+x)^{\frac{1-\kappa}{\kappa}}\left(S(t)+\int R(x)(t+x)^{\frac{1}{\kappa}} d x\right) .
$$

This formula together with the contact transformation from Theorem 6 gives the general solution for the generalized Hunter-Saxton equation (17) in a parametric form:

$$
\begin{aligned}
& \widetilde{u}=\kappa^{2} S^{\prime}(t)+\kappa \int R(x)(t+x)^{\frac{1-\kappa}{\kappa}} d x, \\
& \widetilde{t}=\kappa^{-1} t, \\
& \widetilde{x}=-\kappa\left(S(t)+\int R(x)(t+x)^{\frac{1}{\kappa}} d x\right) .
\end{aligned}
$$

Hence, we obtain the general solution of equation (17) without employing nonlocal transformations.

\section{Discussion}

Cartan's method of equivalence in its different incarnations is a powerful tool in the study of symmetry groups of differential equations. Each of the four approaches discussed has its own advantages and shortcomings. While the first and the second approaches are not universal, the third and the fourth approaches provide an effective technique which is valid irrespective of geometric properties of a given differential equation or of intransitivity of the symmetry pseudogroup. The last approach, the moving coframe method, is applicable to solving the equivalence problems, finding differential invariants and operators of invariant differentiation for classes of differential equations where the power of the infinitesimal method is restricted by complexity of analysis of the defining systems for symmetry generators. Unlike the second and the third methods, the moving coframe method does not require analysis of the defining equations and enables one to find the Maurer-Cartan forms for symmetry pseudo-groups explicitly. The price of its power is lengthy and massive computations. It would be worthwhile to improve the algorithms of the basic steps involved in the method.

We conclude the paper by mentioning evident prospects for the use of Cartan's method in studying structure of symmetry pseudo-groups of differential equations. The directions of future research would include, among others, simplification of solving symmetry classification problems and clarification of the group foliation technique with applications to construction of differentially-invariant solutions. Therefore, the further development and improvement of implementations of Cartan's method would be of great interest and significance for the study of geometry of differential equations. 
[1] Bryant R.L., Griffiths Ph.A., Characteristic cohomology of differential systems (II): conservation laws for a class of parabolic equations, Duke Math. J., 1995, V.78, 531-676.

[2] Bryant R., Griffiths Ph., Hsu L., Hyperbolic exterior differential systems and their conservation laws. I, II, Selecta Math., New Ser., 1995, V.1, 21-112, 265-323.

[3] Bryant R., Griffiths Ph., Hsu L., Toward a geometry of differential equations, in Geometry, Topology \& Physics, Conf. Proc. Lecture Notes Geom. Topology, IV, Cambridge, Internat. Press, 1995, 1-76.

[4] Bluman G.W., Kumei S., Symmetries and differential equations, New York, Springer, 1989.

[5] Cartan É., Sur la structure des groupes infinis de transformations, in Euvres Complètes, Partie II, Vol. 2, Paris, Gauthier-Villars, 1953, 571-714.

[6] Cartan É., Les sous-groupes des groupes continus de transformations, in CEuvres Complètes, Partie II, Vol. 2, Paris, Gauthier-Villars, 1953, 719-856.

[7] Cartan É., La structure des groupes infinis, in CEuvres Complètes, Partie II, Vol. 2, Paris, Gauthier-Villars, 1953, 1335-1384.

[8] Cartan É., Les problèmes d'équivalence, in CEuvres Complètes, Partie II, Vol. 2, Paris, Gauthier-Villars, 1953, 1311-1334.

[9] Cheh J., Olver P.J., Pohjanpelto J., Maurer-Cartan equations for Lie symmetry pseudo-groups of differential equations, J. Math. Phys., 2005, V.46, 023504, 11 pages.

[10] Clelland J.N., Geometry of conservation laws for a class of parabolic partial differential equations, Selecta Math., New Ser., 1997, V.3, N 1, 1-77.

[11] Fels M., The equivalence problem for systems of second order ordinary differential equations, Proc. London Math. Soc., 1995, V.71, 221-240.

[12] Fels M., Olver P.J., Moving coframes. I. A practical algorithm, Acta. Appl. Math., 1998, V.51, $161-213$.

[13] Foltinek K., Quasilinear third-order scalar evolution equations and their conservation laws, Ph.D. Thesis, Duke University, 1996.

[14] Flanders H., Differential forms with applications to the physical sciences, New York - London, Academic Press, 1963.

[15] Gardner R.B., The method of equivalence and its applications. CBMS-NSF regional conference series in applied math., Philadelphia, SIAM, 1989.

[16] Golovin S.V., Group foliation of Euler equations in nonstationary rotationally symmetrical case, in Proceedings of Fifth International Conference "Symmetry in Nonlinear Mathematical Physics" (June 23-29, 2003, Kyiv), Editors A.G. Nikitin, V.M. Boyko, R.O. Popovych and I.A. Yehorchenko, Proceedings of Institute of Mathematics, Kyiv, 2004, V.50, Part 1, 110-117.

[17] Grissom C., Thompson G., Wilkens G., Linearization of second order odes via Cartan's equivalence method, J. Differential Equations, 1989, V.77, N 1, 1-15.

[18] Dryuma V., On the Riemann and Einstein-Weil geometry in theory of the second order ordinary differential equations, math.DG/0104278.

[19] Hsu L., Kamran N., Classification of second order ordinary differential equations admitting Lie groups of fiber-preserving symmetries, Proc. London Math. Soc., Ser. 3, 1989, V.58, N 2, 387-416.

[20] Hunter J.K., Saxton R., Dynamics of director fields, SIAM J. Appl. Math., 1991, V.51, N 6, $1498-1521$.

[21] Ibragimov N.H., Transformation groups applied to mathematical physics, Dordrecht, Reidel, 1985.

[22] Ibragimov N.H., Invariants of hyperbolic equations: solution to Laplace's problem, J. Appl. Mech. Tech. Phys., 2004, V.45, N 2, 11-21.

[23] Johnpillai I.K., Mahomed F.M., Wafo Soh C., Basis of joint invariants for $(1+1)$ linear hyperbolic equations, J. Nonlinear Math. Phys., 2002, V.9, Suppl. 2, 49-59.

[24] Kamran N., Lamb K.G., Shadwick W.F., The local equivalence problem for $y^{\prime \prime}=f\left(x, y, y^{\prime}\right)$ and the Painlevé transcendents, J. Diff. Geometry, 1985, V.22, N 2, 139-150.

[25] Kamran N., Shadwick W.F., A differential geometric characterization of the first Painlevé transcendents, Mathematische Annalen, 1987, V.279, N 1, 117-123.

[26] Kamran N., Shadwick W.F., Équivalence locale des équations aux dérivées partielles quasi lineaires du deixième ordre et pseudo-groupes infinis, Comptes Rendus Acad. Sci. (Paris). Ser. I, 1986, V.303, 555-558.

[27] Kamran N., Contributions to the study of the equivalence problem of Élie Cartan and its applications to partial and ordinary differential equations, Acad. Roy. Belg. Cl. Sci. Mém. Collect $8^{\circ}$ (2), 1989, V.45, N 7.

[28] Krasil'shchik I.S., Lychagin V.V., Vinogradov A.M., Geometry of jet spaces and nonlinear partial differential equations, New York, Gordon and Breach, 1986. 
[29] Laplace P.S., Recherches sur le calcul intégral aux différences partielles, Mémoires de l'Academie Royale de Sciences de Paris, 1777, 341-401; Reprinted in Cuvres Complètes, Vol. 9, Paris, Gauthier-Villars, 1893, 3-68 (English translation, New York, 1966).

[30] Lie S., Gesammelte Abhandlungen, V.1-6, Leipzig, Teubner, 1922 - 1937.

[31] Lisle I.G., Reid G.J., Boulton A., Algorithmic determination of structure of infinite Lie pseudogroups of symmetries of PDEs, in Proc. ISSAC'95, New York, ACM Press, 1995, 1-6.

[32] Lisle I.G., Reid G.J., Geometry and structure of Lie pseudogroups from infinitesimal defining equations, J. Symb. Comp., 1998, V.26, 355-379.

[33] Morozov O.I., Moving coframes and symmetries of differential equations, J. Phys. A: Math. Gen., V.35, N 12, 2965-2977.

[34] Morozov O.I., Symmetries of differential equations and Cartan's equivalence method, in Proceedings of Fifth International Conference "Symmetry in Nonlinear Mathematical Physics" (June 23-29, 2003, Kyiv), Editors A.G. Nikitin, V.M. Boyko, R.O. Popovych and I.A. Yehorchenko, Proceedings of Institute of Mathematics, Kyiv, 2004, V.50, Part 1, 196-203.

[35] Morozov O.I., Contact equivalence problem for linear hyperbolic equations, Proceedings of I.G. Petrovsky's Seminar, 2005, V.25, accepted; math-ph/0406004.

[36] Olver P.J., Applications of Lie groups to differential equations, New York, Springer, 1986.

[37] Olver P.J., Equivalence, invariants, and symmetry, Cambridge, Cambridge University Press, 1995.

[38] Olver P.J., Pohjanpelto J., Moving frames for pseudo-groups. I. The Maurer-Cartan forms, Preprint, University of Minnesota, 2003.

[39] Olver P.J., Pohjanpelto J., Moving frames for pseudo-groups. II. Differential invariants for submanifolds, Preprint, University of Minnesota, 2003.

[40] Olver P.J., Rosenau Ph., Tri-Hamiltonian duality between solitons and solitary wave solutions having compact support, Phys. Rev. E, 1996, V.53, 1900-1906.

[41] Ovsiannikov L.V., Group properties of the Chaplygin equation, J. Appl. Mech. Tech. Phys., 1960, N 3, $126-145$.

[42] Ovsiannikov L.V., Group analysis of differential equations, New York, Academic Press, 1982.

[43] Pavlov M.V., The Calogero equation and Liouville type equations, Theor. and Math. Phys., 2001, V.128, 927-932; nlin.SI/0101034.

[44] Reyes E.G., The soliton content of the Camassa-Holm and Hunter-Saxton equations, in Proceedings of Fourth International Conference "Symmetry in Nonlinear Mathematical Physics" (July 9-15, 2001, Kyiv), Editors A.G. Nikitin, V.M. Boyko and R.O. Popovych, Proceedings of Institute of Mathematics, Kyiv, 2002, V.43, Part 1, 201-208.

[45] Surovikhin K.P., Cartan's exterior forms and computation of the basic group admitted by a given system of differential equations, Moscow Univ. Bulletin, Ser. Math., Mech., 1965, N 6, 70-81 (in Russian).

[46] Tod K.P., Einstein-Weil spaces and third order differential equations, J. Math. Phys., 2000, V.41, 5572-5581. 